\title{
Variables predictoras del compromiso laboral y académico en trabajadores y estudiantes de una universidad peruana ${ }^{1}$
}

\author{
Patricia Martínez-Uribe², Mónica Cassaretto-Bardales³, Mariela Tavera-Palomino ${ }^{4}$ \\ Pontificia Universidad Católica del Perú
}

Recibido: 24/09/2018Ａceptado: 08/11/2019

\begin{abstract}
Resumen
Objetivo. Analizar las variables personales, psicológicas y laborales o académicas que predicen el compromiso en trabajadores (docentes y administrativos) y estudiantes de una universidad peruana. El estudio 1 analiza el compromiso laboral, y el estudio 2, el compromiso académico. Método. En el estudio participaron 512 trabajadores y 1078 estudiantes de una universidad privada de Lima, Perú. A todos se les administró el SF-36, las escalas de Afectividad y Florecimiento que evalúan bienestar, la UWES-9 laboral o académica y la Escala de Eficacia Profesional del MBI para trabajadores y la de Autoeficacia Académica para los estudiantes. Resultados. Las variables predictoras más significativas del compromiso laboral fueron satisfacción con el trabajo $(\beta=0.34$, $p<0.001)$, eficacia profesional $(\beta=0.21, p<0.001)$ y florecimiento $(\beta=0.21, p<0.001)$, y las del compromiso académico, satisfacción con la carrera $(\beta=0.35, p<0.001)$, florecimiento $(\beta=0.33, p<0.001)$ y autoeficacia académica $(\beta=0.19, p<0.001)$. Conclusiones. Las variables predictoras del compromiso fueron similares en ambos estudios. Destacan la satisfacción con el trabajo o la carrera, la autoeficacia y el florecimiento, que es la dimensión del bienestar que supone una vida con sentido, caracterizada por la presencia de vínculos positivos y gratificantes.
\end{abstract}

Palabras clave. Compromiso laboral, compromiso académico, bienestar, autoeficacia.

\section{Predictive Variables of Work and Academic Engagement in Workers and Students of a Peruvian University}

\footnotetext{
Abstract

Objective. To analyze the personal, psychological and labor or academic variables that predict engagement in workers (professors and administrative workers) and students of a Peruvian university. Study 1 analyzes work engagement; Study 2, academic engagement. Method. The participants were 512 workers and 1078 students from a private university in Lima, Peru. All were administered the SF-36, the Affectivity and Flourishing scales

Investigación financiada por la Pontificia Universidad Católica del Perú, como parte del proyecto de investigación sobre Salud y bienestar en el contexto universitario (No. 0333-2016).

2 Doctora en Psicología. Profesora principal del Departamento de Psicología. Dirección de correspondencia: Av. Universitaria 1801, San Miguel, Lima, Perú. Teléfonos: (511)626-2000, anexo 4596; (51)9994-23801. Correo de correspondencia: pmartin@pucp.pe

3 Magíster en Psicología Clínica y de la Salud. Profesora asociada del Departamento de Psicología.

4 Magíster en Psicología Clínica de la Salud. Profesora del Departamento de Psicología.
} 
that assess well-being, the Work or Academic UWES-9 and the scales of Professional Self-efficacy of the MBI for the workers, and the Academic Self-efficacy for students. Results. The predictive variables most significant for work engagement were job satisfaction $(\beta=0.34, p<0.001)$, professional self-efficacy $(\beta=0.021, p<$ $0.001)$ and flourishing $(\beta=0.21, p<0.001)$; for academic engagement: satisfaction with the career $(\beta=0.35, p$ $<0.001)$, flourishing $(\beta=0.33, p<0.001)$ and academic self-efficacy $(\beta=0.19, p<0.001)$. Conclusion. The predictive variables of engagement were similar in both studies. The most important ones were satisfaction with work or career, self-efficacy and flourishing, which is the dimension of well-being that entails a purposeful life, characterized by positive and gratifying social relationships.

Keywords. Work engagement, academic engagement, wellbeing, self-efficacy.

\section{Variáveis preditoras do compromisso laboral e académico em trabalhadores e estudantes de uma universidade peruana}

\section{Resumo}

Escopo. Analisar as variáveis pessoais, psicológicas e laborais ou académicas que predizem o compromisso em trabalhadores (docentes ou administrativos) e estudantes de uma universidade peruana. O estudo 1 analisa o compromisso laboral, e o estudo 2, o compromisso académico. Metodologia. No estudo participaram 512 trabalhadores e 1078 estudantes de uma universidade privada de Lima, Perú. A todos foi administrado o SF-36, as escadas de Afetividade e Floração que avaliam o bem-estar, a UWES-9 laboral ou académica e a Escada de Eficácia Profissional do MBI para trabalhadores e a de Autoeficácia Académica para os estudantes. Resultados. As variáveis preditoras mais significativas do compromisso laboral foram satisfação com o trabalho ( $\beta=0.34$, $p<0.001)$, eficácia profissional $(\beta=0.34, p<0.001)$ e floração $(\beta=0.21, p<0.001)$, e as de compromisso académico, satisfação com a carreira $(\beta=0.35, p<0.001)$, floração $(\beta=0.33, p<0.001)$ e a autoeficácia académica $(\beta=0.19, p<0.001)$. Conclusão. As variáveis preditoras do compromisso foram similares nos dois estudos. Destacam a satisfação com o trabalho ou carreira, a autoeficácia e a floração, que é uma dimensão do bem-estar que supõe uma vida com sentido, caracterizada pela presença de vínculos positivos e gratificantes.

Palavras-chave. Compromisso laboral, compromisso académico, bem-estar, autoeficácia.

\section{Introducción}

El compromiso o engagement es un constructo que ha despertado un creciente interés en la psicología. La preocupación por temas de salud en contextos académicos y laborales, originalmente centrada en los aspectos negativos o desadaptativos, ha dado un giro hacia aquellos aspectos que permiten a las personas encontrar bienestar y realización a través del trabajo o los estudios.

En este contexto surge la presente investigación, como una necesidad de analizar más a fondo este constructo y las variables psicológicas, personales y laborales o académicas que pueden contribuir a su desarrollo. Los estudios originales sobre el compromiso (work engagement) surgen en contextos laborales y posteriormente se amplían a los académicos (academic engagement).
Schaufeli, Martínez, Marques-Pinto, Salanova y Bakker (2002) definen el compromiso como un constructo con componentes actitudinales y comportamentales, que implica entusiasmo, pasión, energía y esfuerzo. Estos estados hacen que la experiencia de trabajar o estudiar sea algo positivo y gratificante. El compromiso, según estos autores, consta de tres componentes: (a) vigor, asociado a altos niveles de esfuerzo, energía y persistencia; (b) dedicación, vinculada al entusiasmo volcado en la actividad; y (c) absorción, que supone la posibilidad de sumergirse en el trabajo o estudios de modo que el tiempo transcurre rápidamente.

Estudios realizados con la Utrech Work Engagement Scale, en diferentes países, muestran asociaciones entre compromiso y diversas variables personales y laborales, generalmente relacionadas con el bienestar, la satisfacción laboral o académica 
y el desempeño, entre otras. Así, Zecca et al. (2015) afirman que las personas comprometidas usualmente son activas, conscientes, emocionalmente estables y extrovertidas; además, en ellas se destaca el rol de las variables personales en su desarrollo.

En contextos laborales, en una investigación con empleados de diversas empresas en España, Acosta, Salanova y Llorens (2012) encontraron que el compromiso se relacionaba positivamente con prácticas organizacionales, en general, saludables, relación en la que cobra importancia la confianza en la organización. Por su parte, Bakker, Schaufeli, Leiter y Taris (2008) realizaron una recopilación de investigaciones sobre el tema y encontraron que el compromiso predice tanto los recursos laborales como los personales de los trabajadores. Los primeros se refieren a retroalimentación, autonomía en el trabajo y adecuada supervisión, mientras que los segundos, a optimismo, autoestima y autoeficacia. Asimismo, consideran el compromiso como un predictor importante del desempeño laboral. Afirman que los empleados comprometidos tienen una ventaja competitiva en las organizaciones, porque tienen alta energía, son entusiastas y están inmersos en el trabajo. Estos hallazgos son similares a los de Moura, Orgambídez y Gonçalves (2014), en Portugal, quienes describen a los empleados comprometidos como más satisfechos y efectivos en su trabajo.

En la misma línea, Durán, Extremera, Montalbán y Rey (2005) analizaron la relación entre compromiso y agotamiento profesional con satisfacción laboral en docentes españoles. En sus resultados destaca la asociación entre cansancio emocional (escala del MBI) y dedicación (UWES) con satisfacción vital. La escala de vigor, por otro lado, es la que mayor relación presenta con satisfacción laboral y satisfacción vital, aunque no las predice. De acuerdo con el modelo de demandas y recursos laborales, Rodríguez, Martínez y Sánchez (2014) explican el bienestar en trabajadores como producto de un balance entre las demandas del trabajo y los recursos personales y organizacionales que generan un mayor compromiso y desempeño más eficiente. Hallaron asociaciones positivas entre satisfacción laboral, compromiso organizacional e involucramiento con el trabajo.

Los estudios de Seppälä et al. (2009) asocian el compromiso a la salud física y mental, a un sentimiento de bienestar general. Encontraron asociaciones con motivación intrínseca y autoeficacia. Estos hallazgos coinciden con los de Mills, Culbertson y Fullagar (2012), quienes, además, la asociaron con necesidades de logro, afecto positivo y satisfacción laboral. Entre las variables personales, Simbula, Guguelmi, Schaufeli y Depolo (2013) hallaron asociaciones con salud mental, y menor incidencia de depresión, ansiedad y estrés. Estos investigadores describen, asimismo, una espiral positiva entre el compromiso y la autoeficacia que resulta en una mayor satisfacción laboral y productividad.

En contextos académicos, Paradnikè y Bandzevičienè (2016) analizaron la asociación entre el compromiso académico de estudiantes universitarios y los recursos para adaptarse a la carrera, que suponen preocupación por los estudios, sensación de control, curiosidad y confianza. Encontraron que preocupación y confianza son predictores del compromiso.

La relación entre el compromiso y el rendimiento académico en diferentes contextos parece confirmar una asociación positiva. En esta línea, el estudio de García, Labajos y Fernández (2014) con estudiantes de Enfermería en España, mostró correlaciones positivas entre ambos constructos, de manera similar a lo hallado por Caballero, Hederich y García (2015) con estudiantes universitarios en Colombia. En este, el compromiso resultó ser un buen predictor de buen desempeño académico, y destacaron que las tres dimensiones del compromiso correlacionaban también positivamente con satisfacción con el estudio. Por su parte, Gómez et al. (2015) encontraron que los logros académicos de estudiantes de Medicina correlacionan con el compromiso, y que aquellos con mayor compromiso muestran un elevado bienestar.

Los estudios de Ouweneel, Le Blanc y Schaufeli (2012, 2013) con estudiantes universitarios, confirman la relación del compromiso, en particular de la dimensión de vigor, con desempeño académico. Aquí, la autoeficacia impacta positivamente en la motivación y, con ello, en el compromiso y el desempeño. Finalmente, relacionan el compromiso con recursos personales de los estudiantes y con el predominio de emociones positivas.

En esta misma línea, Uludag (2016) analizó el efecto del compromiso y del afecto positivo en el desempeño académico de estudiantes 
universitarios, y encontró que tanto el compromiso como el afecto positivo tienen un efecto positivo y significativo en el desempeño académico. Sin embargo, existe creciente evidencia de dificultades en torno a la salud mental de docentes y estudiantes universitarios, descritos como una población vulnerable para desarrollar trastornos emocionales de diversa índole (Antúnez y Vinet, 2013; MarencoEscuderos y Ávila-Toscano, 2016).

Considerando los estudios reseñados previamente sobre el compromiso en diferentes ámbitos, parece clara la asociación del constructo con variables positivas como bienestar, satisfacción y autoeficacia, entre otros. Es así que el objetivo del presente estudio fue analizar los predictores personales, psicológicos y laborales o académicos del compromiso, tanto en trabajadores como en estudiantes de una universidad peruana. Por esta razón, estudiar el compromiso puede contribuir a fortalecer aquellos aspectos que redunden en un mayor bienestar en esta población. El estudio 1 se centra en el compromiso laboral de docentes y personal administrativo, y el estudio 2, en el compromiso académico de estudiantes.

\section{Estudio 1}

El objetivo de este estudio fue analizar variables personales, psicológicas y laborales que podrían predecir el compromiso laboral en docentes y administrativos que laboran en una universidad privada de Lima, Perú. Las variables psicológicas consideradas fueron eficacia profesional, bienestar (afectividad y florecimiento) y salud percibida. Las variables personales fueron sexo y edad. En tanto las variables laborales fueron satisfacción con el trabajo, tiempo de servicios y condición laboral, esto es si es docente o administrativo, nombrado o contratado en la institución.

\section{Método}

\section{Participantes.}

En el estudio participaron 512 trabajadores de una universidad privada de Lima, Perú, con edades entre los 23 y los 78 años $(M=44.78, D E=12.21)$, siendo el $51.2 \%$ mujeres, y el $48.8 \%$ varones; todos ellos con un mínimo de dos años laborando a tiempo completo en la institución. Los 226 docentes procedían de diferentes departamentos académicos, de los cuales, el $96.2 \%$ estaban nombrados, y 286 trabajadores administrativos de distintas unidades, ejecutivos, asistentes y técnicos, de los cuales, el $69 \%$ se encontraban nombrados.

\section{Instrumento.}

Para medir el compromiso laboral (engagement) se utilizó la versión abreviada de la Utrecht Work Engagement Scale (UWES) de Schaufeli et al. (2002), que consta de nueve ítems distribuidos en tres dimensiones: vigor, dedicación y absorción. Los ítems se presentan en una escala Likert de siete puntos, desde Nunca hasta Siempre, todos los días. Se utilizó la versión en español propuesta por los mismos autores, quienes reportan estudios con 12.631 participantes de diferentes países, que confirman la estructura trifactorial del instrumento, tanto para la versión completa de 17 ítems como para la abreviada. Los coeficientes alfa reportados para las tres dimensiones de la prueba oscilan entre 0.72 y 0.84 , y 0.90 para la prueba total (Schaufeli y Bakker, 2003). La UWES ha sido utilizada y validada en distintos idiomas y países, y ha demostrado adecuadas propiedades psicométricas. En Perú, Flores, Fernández, Juárez, Merino y Guimet (2015) realizaron la validación del instrumento con un análisis factorial del mismo y alfas entre 0.61 y 0.77 , confirmándose su estructura trifactorial. Resultados muy similares tuvieron Sánchez-Cardona, Rodríguez-Montalbán, Toro-Alfonso y MorenoVelázquez (2016) en México, con coeficientes alfa entre 0.82 y 0.88 para las tres dimensiones. En el presente estudio, el coeficiente de confiabilidad alfa de Cronbach para la prueba total fue de 0.90, y para las áreas, fueron 0.77 para vigor, 0.89 para dedicación y 0.74 para absorción.

En cuanto a la eficacia profesional, esta fue evaluada a través de la Escala de Eficacia Profesional del Maslach Burnout Inventory en su forma general (MBI-GS), creada por Maslach, Jackson y Leiter en 1996. Si bien el instrumento fue creado con el fin de evaluar el síndrome de agotamiento profesional, es frecuente su uso independientemente de la evaluación global del agotamiento profesional. Diversos estudios confirman adecuadas propiedades psicométricas en el instrumento y en la escala. Los coeficientes de confiabilidad reportados para la Escala de Eficacia Profesional en varios estudios son de 0.81 (Oramas, González y Vergara, 2007), 0.85 
(Moreno-Jiménez, Rodríguez-Carvajal y Escobar, 2001) y 0.77 (Fernández-Arata, Juárez y Merino, 2015). En la presente investigación, el coeficiente de confiabilidad para dicha escala fue de 0.83 .

El bienestar fue evaluado mediante dos escalas. El Spane, que mide afecto positivo y negativo, y la Escala de Florecimiento, que evalúa la dimensión eudaimónica del bienestar, es decir, la percepción de una vida plena, con significado y vínculos positivos y gratificantes (Diener et al., 2010). Este instrumento consta de seis adjetivos positivos y seis negativos dispuestos en una escala Likert de cinco puntos, y la Escala de Florecimiento, de ocho ítems en una escala Likert de siete puntos. Los estudios iniciales del autor reportan coeficientes alfa de 0.87 para la Escala de Florecimiento, y 0.87 y 0.81 para afecto positivo y negativo, respectivamente. Las escalas han sido validadas en diferentes contextos con muy buenos resultados. En Perú, Cassaretto y Martínez (2017) realizaron la traducción y validación de las escalas con 656 estudiantes, reportando coeficientes alfa de 0.89 para florecimiento, $y$ para las escalas de afecto positivo y negativo, 0.91 y 0.87 , respectivamente. En el presente estudio, las confiabilidades obtenidas fueron de 0.86 para afecto positivo, 0.82 para afecto negativo y 0.87 para la Escala de Florecimiento.

Para medir la salud percibida se utilizó el Cuestionario de Salud SF-36 desarrollado por Ware y Gandek (1998). Consta de 36 ítems que describen ocho dimensiones de salud que se agrupan en dos componentes, salud física y salud mental. La versión en español fue desarrollada por Alonso, Prieto y Antó (1995), con la participación de 15 países; los coeficientes alfa reportados para todas las dimensiones superaron el 0.7 esperado. Lo mismo encontraron Vilagut et al. (2005) en un metaanálisis de 79 artículos, aplicando el instrumento. Ellos reportaron coeficientes de confiabilidad superiores a 0.7 en el $96 \%$ de artículos. Los coeficientes alfa para las ocho escalas en el grupo evaluado en esta investigación fluctuaron entre 0.77 y 0.83 . Los componentes de salud física y salud mental usados en la presente investigación presentaron un alfa de 0.64 y 0.75 , respectivamente.

Adicionalmente, se incluyó una ficha de datos sociodemográficos y preguntas sobre el nivel de satisfacción laboral en una escala Likert de cinco puntos, así como preguntas relativas a su condición laboral y tiempo de servicio en la institución.

\section{Procedimiento.}

El proyecto de investigación del que se desprenden los presentes estudios fue revisado y aprobado por el Comité de Ética de la Investigación de la Pontificia Universidad Católica del Perú (Dictamen 0231-2015/CEI-PUCP).

Luego de las autorizaciones respectivas, se siguieron dos procedimientos distintos para la convocatoria de docentes y personal administrativo. A los primeros se les enviaron cartas personales invitándolos a participar de la investigación. A todos aquellos que aceptaron participar, los investigadores se acercaron a sus oficinas con los cuadernillos de pruebas, les explicaron los objetivos del estudio y se les hizo entrega del consentimiento informado, en el que se explicaba que se trataba de una participación voluntaria y anónima y que podrían suspender la aplicación en el momento en que lo desearan. Asimismo, se explicó que los datos serían procesados de manera conjunta y que no habría resultados individuales. Los protocolos fueron colocados en un ánfora sellada, donde se iban acumulando hasta completar el número esperado. Para la convocatoria del personal administrativo, se recurrió a las unidades con mayor número de colaboradores y se los invitó a participar del estudio en uno de los ambientes de la unidad. Luego de las explicaciones respectivas, similares a las de los docentes, aquellos que aceptaron participar completaron el consentimiento informado y los protocolos, los cuales fueron colocados en sobres cerrados. Los grupos fueron de entre cinco y 12 personas. Esta etapa tomó ocho meses y se concluyó en mayo de 2017.

\section{Analisis de datos.}

Los datos obtenidos fueron procesados mediante el SPSS v. 24. Luego de la depuración de la base, se analizó la confiabilidad de los instrumentos mediante el coeficiente alfa de Cronbach. En todos los casos, los coeficientes fueron aceptables, por lo que se procedió al análisis de las correlaciones mediante el coeficiente $r$ de Pearson. Posteriormente, se realizó un análisis de regresión mediante el método de mínimos cuadrados, con compromiso como variable dependiente. 


\section{Resultados}

En la tabla 1 se presentan las correlaciones entre el compromiso y sus tres dimensiones con todas las variables analizadas. En todos los casos se observan correlaciones significativas, con excepción de la variable sexo.

Tabla 1

Correlaciones entre compromiso laboral y variables psicológicas, sociodemográficas y laborales

\begin{tabular}{lcccc}
\hline Variables & Compromiso & Vigor & Dedicación & Absorción \\
\hline Variables psicológicas & & & & \\
$\quad$ Spane: Balance afectivo & $0.50^{* *}$ & $0.55^{* *}$ & $0.50^{* *}$ & $0.30^{* *}$ \\
$\quad$ FLS: Florecimiento & $0.54^{* *}$ & $0.55^{* *}$ & $0.54^{* *}$ & $0.38^{* *}$ \\
SF-36: C. Salud física & $0.28^{* *}$ & $0.31^{* *}$ & $0.27^{* *}$ & $0.20^{* *}$ \\
SF-36: C. Salud mental & $0.45^{* *}$ & $0.51^{* *}$ & $0.43^{* *}$ & $0.28^{* *}$ \\
$\quad$ MBI: Eficacia profesional & $0.50^{* *}$ & $0.48^{* *}$ & $0.53^{* *}$ & $0.36^{* *}$ \\
Variables sociodemográficas & & & & \\
$\quad$ Sexo & -0.08 & $-0.11^{*}$ & -0.09 & -0.01 \\
$\quad$ Edad & $0.30^{* *}$ & $0.30^{* *}$ & $0.29^{* *}$ & $0.23^{* *}$ \\
Variables laborales & & & & \\
$\quad$ Satisfacción con el trabajo & $0.55^{* *}$ & $0.50^{* *}$ & $0.59^{* *}$ & $0.40^{* *}$ \\
$\quad$ Tiempo de servicio & $0.22^{* *}$ & $0.22^{* *}$ & $0.23^{* *}$ & $0.15^{* *}$ \\
$\quad$ Grupo (Docentes/administrativos) & $-0.15^{* *}$ & $-0.13^{* *}$ & $-0.16^{* *}$ & $-0.13^{* *}$ \\
\hline
\end{tabular}

Nota. Sexo $(0=$ varones, $1=$ mujeres $)$, Grupo $(0=$ docente, $1=$ administrativo $)$

${ }^{*} p<0.05,{ }^{* *} p<0.01$,

Fuente: elaboración propia.

En el análisis de regresión (tabla 2) se obtuvo un $R^{2}$ de $0.54(F[10.441]=51.32, p<0.001)$, donde, de acuerdo con el criterio de Cohen, se evidencia un efecto grande de las variables estudiadas en el compromiso (Ellis, 2010). Las variables con mayor efecto sobre el compromiso fueron florecimiento, eficacia profesional y satisfacción con el trabajo. La edad y el balance positivo de afectos también tuvieron un efecto, aunque menor.

Tabla 2

Análisis de regresión de compromiso laboral como variable dependiente

\begin{tabular}{lccccccc}
\hline & $B$ & $\mathrm{SE}_{\beta}$ & $\beta$ & $t$ & $p$ & Parcial & VIF \\
\hline (Constante) & -2.45 & 1.22 & & -2.01 & 0.046 & & \\
Satisfacción con el trabajo & 1.11 & 0.12 & 0.34 & 9.38 & 0.000 & 0.41 & 1.22 \\
MBI: Eficacia profesional & 0.70 & 0.13 & 0.21 & 5.55 & 0.000 & 0.26 & 1.43 \\
FLS: & 0.11 & 0.02 & 0.21 & 4.93 & 0.000 & 0.23 & 1.75 \\
Florecimiento & & & & & & & \\
Edad & 0.04 & 0.01 & 0.17 & 3.31 & 0.001 & 0.16 & 2.51 \\
Spane: Balance afectivo & 0.04 & 0.02 & 0.10 & 2.18 & 0.030 & 0.10 & 2.17 \\
SF-36: C. Salud mental & 0.01 & 0.01 & 0.06 & 1.17 & 0.244 & 0.06 & 2.26 \\
Sexo & 0.14 & 0.18 & 0.03 & 0.80 & 0.427 & 0.04 & 1.09 \\
SF-36: C. Salud física & 0.00 & 0.01 & 0.02 & 0.50 & 0.614 & 0.02 & 1.43 \\
Tiempo de servicios & 0.00 & 0.09 & 0.00 & 0.05 & 0.958 & 0.00 & 1.95 \\
Grupo (Doc/Adm) & -0.04 & 0.21 & -0.01 & -0.18 & 0.859 & -0.01 & 1.54 \\
\hline
\end{tabular}

Nota. Sexo $(0=$ varones, $1=$ mujeres $)$, Grupo $(0=$ docente, $1=$ administrativo $)$

Fuente: elaboración propia. 


\section{Discusión}

Es importante señalar la presencia de correlaciones positivas y significativas entre el compromiso y todas las variables psicológicas y laborales estudiadas. Las correlaciones más altas se encontraron entre compromiso y eficacia profesional, florecimiento, la cual es una dimensión de sentido del bienestar, y predominio de los afectos positivos sobre los negativos, además de satisfacción con el trabajo. Estos resultados van en el mismo sentido que lo reportado en otros estudios (Mills et al., 2012; Rodríguez et al., 2014; Seppälä et al., 2009). Esto supone que las personas comprometidas, capaces de involucrarse en el trabajo, se sienten a gusto con lo que hacen, se sienten realizadas y capaces de desempeñar sus actividades exitosamente. Hasta aquí no es posible determinar cuáles variables determinan qué, simplemente se puede afirmar que existen asociaciones importantes entre los constructos.

La única variable estudiada que no presentó correlación con compromiso fue el sexo, lo que también va en el sentido esperado. No hay evidencias de mayores diferencias en el compromiso de hombres y mujeres en las investigaciones sobre el constructo. La única asociación se presentó en vigor, que se elevó ligeramente en el grupo de hombres.

El análisis de regresión permitió profundizar en estos datos y proponer una dirección, asumiendo el compromiso como la variable dependiente, que constituye el objetivo central de esta investigación. El nivel de satisfacción laboral reportado por los trabajadores, resultó ser la principal variable predictora del compromiso, seguida por eficacia profesional y florecimiento. Estas tres variables en conjunto son las que determinan el compromiso laboral. Sentirse satisfecho y capaz en y con el trabajo que se realiza, se convierte en una fuente de realización personal que potencia el compromiso. Se formaría así una espiral positiva, como describe Simbula et al. (2013), que incrementa a su vez la productividad de los trabajadores (Bakker et al., 2008; Moura et al., 2014).

Además de las variables descritas, la edad también contribuyó significativamente a elevar el nivel de compromiso, lo mismo que el predominio de afectos positivos sobre los negativos. Lo primero dio cuenta de una mayor estabilidad general en diversos aspectos de la vida, conforme aumenta la edad, que no está ligada necesariamente a aspectos laborales, los cuales se expresarían en el tiempo de servicios, que en este caso no resultó un predictor del compromiso. De otro lado, el predominio de afectos positivos va en el mismo sentido de una sensación agradable y placentera vinculada al trabajo, más fuerte que los inconvenientes o problemas que puedan encontrarse en el mismo. Este resultado va también en la línea de lo descrito por Mills et al. (2012), quienes encontraron una asociación positiva entre el compromiso y el afecto positivo.

Si bien existe una correlación significativa entre los componentes de salud física y mental, de manera similar a lo reportado por Seppälä et al. (2009) y Simbula et al. (2013), estos no resultaron ser predictores del compromiso en el grupo estudiado. Es muy posible que la percepción de una buena salud física y mental estuviera asociada más bien al sentimiento de bienestar general que presentaron aquellos que se sienten comprometidos con su trabajo.

\section{Estudio 2}

\section{Método}

El objetivo de este estudio fue analizar variables personales, psicológicas y académicas que podrían predecir el compromiso académico en estudiantes de una universidad privada de Lima, Perú. Las variables psicológicas consideradas fueron autoeficacia, bienestar (afectividad y florecimiento) y salud percibida. Las variables personales fueron sexo y edad, y entre las académicas, se consideraron satisfacción con la carrera elegida y con el rendimiento.

\section{Participantes.}

Participaron 1078 estudiantes de una universidad privada de Lima, con edades entre los 16 y los 30 años, y una media de $19.97(D E=2.40)$, que se encontraban cursando carreras de pregrado de diferentes facultades -ingenierías, humanidades, derecho y educación-. El $56.6 \%$ eran varones y el $43.4 \%$, mujeres. El $72.1 \%$ nacido en Lima y los demás en provincias. 


\section{Instrumentos.}

Para medir el compromiso académico, se usó la versión académica y abreviada de la Utrech Work Engagement Scale (UWES-S), compuesta por nueve ítems, que son los mismos de la versión original; solo se modificó el contenido de los ítems relativos al contexto académico en lugar del laboral (Schaufeli et al., 2002). Las propiedades psicométricas del instrumento han sido descritas en el estudio 1. Entre los estudios con la versión académica de la UWES se encuentra el de Medrano, Moretti y Ortiz (2015), en el que se confirma la estructura de tres factores con coeficientes alfa entre 0.75 y 0.84 para las dimensiones, similar a los hallazgos de Vizoso y Arias (2016), con coeficientes alfa entre 0.65 y 0.85. El coeficiente alfa de Cronbach hallado en este estudio para la prueba total fue de 0.92, y para las escalas, de 0.81 para vigor, 0.85 para dedicación y 0.81 para absorción.

Con respecto a la salud percibida, se usó el SF-36, que fue el mismo instrumento usado en el estudio 1 con trabajadores, en el que se presentó la información psicométrica del instrumento. En el presente estudio, los coeficientes de confiabilidad oscilaron entre 0.75 y 0.86 para las ocho escalas. En el componente de salud física se obtuvo un alfa de 0.63, y para el componente de salud mental, de 0.72 , que son los que se usaron en la presente investigación.

El bienestar, al igual que en el estudio 1, se evaluó con el Spane y la Escala de Florecimiento. Los datos de la validación de las escalas son los mismos del primer estudio. Los coeficientes de confiabilidad obtenidos para la presente investigación fueron de 0.90 para afecto positivo, 0.85 para afecto negativo y 0.91 para florecimiento.

Por último, para medir autoeficacia académica se utilizó la Escala de Autoeficacia Percibida Específica de Situaciones Académicas (EAPESA; Palenzuela, 1983), que se trata de una medida unidimensional de la autoeficacia contextualizada en el ámbito académico. Consta de 10 ítems con cuatro opciones de respuesta, desde nunca hasta siempre. La validación realizada por el autor confirma la estructura unifactorial del instrumento y un alfa de 0.91 con una muestra de 739 estudiantes. Los estudios de García-Fernandez et al. (2010, 2016) confirman estos hallazgos con coeficientes alfa de 0.89 para España y 0.83 para Chile. En el presente estudio, la escala alcanzó un coeficiente de confiabilidad de 0.91 .

Adicionalmente, se recogió información sociodemográfica y se incluyeron preguntas sobre el nivel de satisfacción de los estudiantes con su carrera y con su rendimiento en la universidad, a través de una escala Likert de cinco puntos.

\section{Procedimiento.}

De modo similar a lo expuesto en el estudio 1, se tuvieron presentes todos los resguardos éticos necesarios para la aplicación de los cuestionarios, los cuales fueron administrados de manera grupal en aulas al finalizar las clases, a todos aquellos estudiantes que voluntariamente accedieron a participar. Ellos firmaron el consentimiento informado y respondieron los cuestionarios de manera anónima. Los grupos evaluados fueron de entre 15 y 30 estudiantes.

\section{Análisis de datos.}

El análisis de los datos siguió los mismos procedimientos descritos en el estudio 1.

\section{Resultados}

Las correlaciones entre compromiso académico y las demás variables estudiadas se presentan en la tabla 3 , donde se observa que las variables psicológicas y académicas se encuentran significativamente asociadas al compromiso, lo que no ocurre con sexo y edad. 
Tabla 3

Correlaciones entre compromiso académico y variables psicológicas, sociodemográficas y académicas

\begin{tabular}{|c|c|c|c|c|}
\hline Variables & Compromiso & Vigor & Dedicación & Absorción \\
\hline \multicolumn{5}{|l|}{ Variables psicológicas } \\
\hline Spane: Balance afectivo & $0.45^{* *}$ & $0.41^{* *}$ & $0.43^{* *}$ & $0.38^{* *}$ \\
\hline FLS: Florecimiento & $0.58^{* *}$ & $0.47^{* *}$ & $0.57^{* *}$ & $0.52^{* *}$ \\
\hline SF-36: C. Salud física & $0.15^{* *}$ & $0.13^{* *}$ & $0.16^{* *}$ & $0.13^{* *}$ \\
\hline SF-36: C. Salud mental & $0.30^{* *}$ & $0.26^{* *}$ & $0.30^{* *}$ & $0.24 * *$ \\
\hline Autoeficacia académica & $0.45^{* *}$ & $0.37^{* *}$ & $0.42^{* *}$ & $0.42^{* *}$ \\
\hline \multicolumn{5}{|l|}{ Variables sociodemográficas } \\
\hline Sexo & 0.03 & $0.07^{*}$ & 0.02 & 0.00 \\
\hline Edad & 0.06 & $0.07^{*}$ & 0.04 & 0.04 \\
\hline \multicolumn{5}{|l|}{ Variables académicas } \\
\hline Satisfacción con la carrera & $0.55^{* *}$ & $0.40^{* *}$ & $0.63^{* *}$ & $0.45^{* *}$ \\
\hline Satisfacción con rendimiento & $0.36^{* *}$ & $0.32^{* *}$ & $0.35^{* *}$ & $0.32^{* *}$ \\
\hline
\end{tabular}

${ }^{*} p<0.05,{ }^{* *} p<0.01$

Fuente: Elaboración propia.

En la tabla 4 se presenta el análisis de regresión, donde se obtiene un $R^{2}$ de $0.51(F[9.987]=113.56$, $p>0.000)$, y nuevamente, se observa un efecto grande de las variables analizadas sobre el compromiso académico. Destacan la satisfacción con la carrera, florecimiento, autoeficacia académica $y$, en menor medida, balance afectivo y sexo.

Tabla 4

Análisis de regresión de compromiso académico como dependiente

\begin{tabular}{lccccccc}
\hline & $B$ & $\mathrm{SE}_{\beta}$ & $\beta$ & $t$ & $p$ & Parcial & VIF \\
\hline (Constante) & -2.62 & 0.92 & & -2.85 & 0.004 & & \\
Satisfacción con la carrera & 1.28 & 0.09 & 0.35 & 14.11 & 0.000 & 0.41 & 1.26 \\
FLS: Florecimiento & 0.13 & 0.01 & 0.33 & 10.92 & 0.000 & 0.33 & 1.79 \\
Autoeficacia académica & 1.09 & 0.15 & 0.19 & 7.22 & 0.000 & 0.22 & 1.44 \\
Spane: Balance afectivo & 0.04 & 0.01 & 0.09 & 2.53 & 0.012 & 0.08 & 2.56 \\
Sexo & 0.40 & 0.15 & 0.06 & 2.59 & 0.010 & 0.08 & 1.10 \\
Satisfacción con rendimiento & 0.15 & 0.09 & 0.04 & 1.58 & 0.113 & 0.05 & 1.34 \\
Edad & 0.01 & 0.03 & 0.00 & 0.16 & 0.870 & 0.01 & 1.03 \\
SF-36: C. Salud física & -0.01 & 0.01 & -0.03 & -1.03 & 0.302 & -0.03 & 1.38 \\
SF-36: C. Salud mental & -0.01 & 0.01 & -0.04 & -1.22 & 0.224 & -0.04 & 2.28 \\
\hline
\end{tabular}

Nota. Sexo $(0=$ varones, $1=$ mujeres $)$

Fuente: elaboración propia. 


\section{Discusión}

Los hallazgos en el grupo de estudiantes fueron similares a los del grupo de trabajadores. Nuevamente, aquí todas las variables psicológicas y académicas correlacionaron con el compromiso. En el análisis de regresión, la satisfacción con la carrera elegida resultó ser el principal determinante de compromiso, lo mismo que el florecimiento, asociado a una sensación de realización personal. Esto confirma la importancia de realizar una adecuada elección vocacional, ya que en la medida en que los jóvenes sienten que están en el camino correcto para ellos, el compromiso y el bienestar se incrementan, lo mismo que la percepción de autoeficacia. Los estudios de Caballero et al. (2015), Gómez et al. (2015), Ouweneel et al. (2013) y Uludag (2016) van en un sentido similar, ya que encuentran asociaciones con la satisfacción con los estudios, el bienestar, la autoeficacia y los afectos o emociones positivas, respectivamente.

La ausencia de correlaciones entre compromiso y edad reveló que se trataba de un grupo bastante homogéneo. Todos pertenecían a la misma universidad, con un rango de edad similar, lo que hacía que compartieran preocupaciones, intereses e incluso metas, proyectos y valores, y también explicaría la ausencia de correlaciones con la variable sexo. Si bien en este caso, en el análisis de regresión, aparecía como una variable significativa, su aporte al modelo fue muy bajo y probablemente más asociado al ligero incremento de la subescala de vigor en el grupo de mujeres.

De otro lado, si bien se dio una correlación significativa entre la satisfacción con el rendimiento y el compromiso, no alcanzó a ser una variable predictora del mismo. Es muy probable que el buen rendimiento en los estudios se asociara con un mayor compromiso, por eso la correlación, pero es claro que no lo determinaba.

Los componentes de salud percibida tampoco determinaron los niveles de compromiso, aunque también correlacionaran con él. Sentirse física y mentalmente bien va de la mano con un sentido de bienestar y compromiso, pero sentirse bien no hace que los estudiantes, en este caso, se involucren más en sus estudios.

\section{Discusión general}

Los dos estudios llevados a cabo pretendían aportar información sobre aquellas variables que contribuyen a desarrollar un mayor sentido de compromiso, ya sea con el trabajo o los estudios. Los resultados mostraron que en ambos casos el sentirse satisfecho, con el trabajo o la carrera elegida, el sentirse eficaz en las tareas que se desarrollan y el sentir cierto grado de realización personal, florecimiento, fueron los principales predictores del compromiso. Complementa a estas tres variables el predominio de los afectos positivos sobre los negativos, que conjuntamente con el florecimiento están asociadas con un sentimiento de bienestar integral que abarca tanto la sensación de placer como la de sentido.

Asimismo, los hallazgos revelaron que en ambos estudios, la salud percibida se encuentra claramente asociada con un mayor compromiso, pero no lo determina o predice. Resulta interesante anotar que, sin embargo, la correlación entre ambos constructos fue mayor en el grupo de trabajadores que en el de estudiantes. Probablemente, la edad desempeñó un rol importante, ya que es en el grupo de adultos trabajadores en el que los niveles de compromiso se incrementan con la edad.

Estos resultados van en el mismo sentido que los estudios descritos previamente, los cuales subrayan la asociación entre el compromiso y las variables positivas, como bienestar, autoeficacia o satisfacción, como lo encontrado por Zecca et al. (2015), Gómez et al. (2015), Uludag (2016) y Moura et al. (2014). Estos estudios se centran particularmente en asociaciones, en las que no es posible determinar el sentido de las mismas; es decir, ¿es el bienestar o satisfacción el que predice el compromiso?, o ¿es el compromiso el que predice el bienestar o satisfacción? En el presente estudio se propuso analizar estas variables en función del compromiso, que es la variable central del mismo, y los resultados mostraron un posible sentido para las asociaciones descritas. Sin embargo, no se excluye que en un estudio con bienestar o satisfacción como variables dependientes pudieran darse resultados similares, siendo, en este caso, el compromiso uno de los predictores de bienestar. El tema queda abierto para futuras investigaciones. 
Un dato adicional, que resulta interesante discutir, tiene que ver con la conveniencia de trabajar con el compromiso con sus tres componentes o como medida global y única. Al respecto, numerosos estudios indican que el modelo trifactorial de la UWES es el que se ajusta mejor; sin embargo, también se indica que un modelo unifactorial también es posible (Pujol y Arraigada, 2018). En el presente estudio, lo que pudo observarse es que si bien los factores proporcionan algunos matices en el análisis de correlaciones, los resultados son muy similares, lo que apoyaría la posibilidad de trabajar el instrumento de ambas formas.

\section{Referencias}

Acosta, H., Salanova, S., \& Llorens, S. (2012). How Organizational Practices Predict Team Work Engagement: The Role of Organizational Trust. Ciencia y Trabajo, año 14(número especial), 7-15. Recuperado de https://www.researchgate. net/publication/235991068_How organizational_practices_predict_team_work_ engagement_The_role_of_organizational_trust

Alonso, J., Prieto, L. y Antó, J. (1995). La versión española del SF-36 Health Survey (Cuestionario de Salud SF-36): Un instrumento para la medida de los resultados clínicos. Medicina Clínica, 104(20), 771-776.

Antúnez, Z. y Vinet, E. (2013). Problemas de salud mental en estudiantes de una universidad regional chilena. Revista Médica de Chile, 141(2), 209-216. http://dx.doi.org/10.4067/ S0034-98872013000200010

Bakker, A., Schaufeli, W., Leiter, M. \& Taris, T. (2008). Work Engagement: An Emerging Concept in Occupational Health Psychology. Work \& Stress. An International Journal of Work, Health \& Organizations, 22(3), 187-200. doi: 10.1080/02678370802393649

Caballero, C., Hederich, C. y García, A. (2015). Relación entre burnout y engagement académicos con variables sociodemográficas y académicas. Psicología desde el Caribe, 32(2), 254-267. http://dx.doi.org/10.14482/ psdc.32.2.5742

Cassaretto, M. y Martínez, P. (2017). Validación de las escalas de bienestar: Florecimiento y afec- tividad. Pensamiento Psicológico, 15(1), 19-31. Recuperado de https://revistas.javerianacali. edu.co/index.php/pensamientopsicologico/article/view/1255

Diener, E., Wirtz, D., Tov, W., Kim-Prieto, C., Choi, DW., Oishi, S. \& Biswas-Diener, R. (2010). New Well-Being Measures: Short Scales to Assess Flourishing and Positive and Negative Feelings. Social Indicators Research, 97(2), 143-156. https://doi.org/10.1007/s11205-0099493-y

Durán, M., Extremera, N., Montalbán, F. y Rey, L. (2005). Engagement y Burnout en el ámbito docente: Análisis de sus relaciones con la satisfacción laboral y vital en una muestra de profesores. Revista de Psicología del Trabajo y de las Organizaciones, 21(1-2), 145-158. Recuperado de https://www.redalyc.org/ articulo.oa?id=231317039009

Ellis, P. D. (2010). The Essential Guide to Effect Sizes: Statistical Power, Meta-Analysis, and the Interpretation of Research Results. Cambridge: Cambridge University Press.

Fernández-Arata, M., Juárez, A. y Merino, C. (2015). Análisis estructural e invarianza de medición del MBI-GS en trabajadores peruanos. Liberabit, 21(1), 9-20. Recuperado de http://ojs3.revistaliberabit.com/index.php/Liberabit/article/ view/282

Flores, C., Fernández, M., Juárez, A., Merino, C. y Guimet, M. (2015). Entusiasmo por el trabajo (engagement): un estudio de validez en profesionales de la docencia en Lima, Perú. Liberabit, 21(2), 195-206. Recuperado de http:// ojs3.revistaliberabit.com/index.php/Liberabit/ article/view/261

García-Fernández, J., Inglés-Saura, C., Torregrosa, M., Ruiz-Esteban, C., Díaz-Herrero, A., PérezFernández, E. y Martínez-Monteagudo, M. (2010). Propiedades psicométricas de la Escala de Autoeficacia Percibida específica de situaciones académicas en una muestra de estudiantes españoles de Educación Secundaria Obligatoria. European Journal of Education and Psychology, 2(1), 61-74. doi:10.30552/ejep. v3i1.51

García-Fernández, J., Inglés-Saura, C., Vincent, M., Gonzálvez C., Lagos-San Martín, N. y Pérez- 
Sánchez, A. (2016). Relación entre autoeficacia y autoatribuciones académicas en estudiantes chilenos. Universitas Psychologica, 15(1), 1524. doi:10.11144/Javeriana.upsy15-1.raaa

García, J., Labajos, M. y Fernández, F. (2014). Estudio de los estresores en los prácticum de Grado en Enfermería. Rol de Enfermería, 37(7-8) 488494. Recuperado de https://medes.com/publication/93392

Gómez, P., Pérez, C., Parra, P., Ortiz, L., Matus, O., McColl, P., Torres, G. y Meyer, A. (2015). Relación entre el bienestar y el rendimiento académico en alumnos de primer año de Medicina. Revista Médica de Chile, 143(7), 930-937. doi:10.4067/S0034-98872015000700015

Marenco-Escuderos, A. y Ávila-Toscano, J. (2016). Burnout y problemas de salud mental en docentes: Diferencias según características demográficas y sociolaborales. Psychologia: Avances de la Disciplina, 10(1), 91-100. Recuperado de http://www.scielo.org.co/pdf/ psych/v10n1/v10n1a09.pdf

Maslach, C., Jackson, S. y Leiter; M. (1996). Maslach Burnout Inventory. Palo Alto, CA: Consulting Psychologist Press.

Medrano, L., Moretti, L y Ortiz, A. (2015). Medición del engagement académico en estudiantes universitarios. Revista Iberoamericana de Diagnóstico y Evaluación Psicológica, 40(1), 114-123. Recuperado de http://www.redalyc. org/articulo.oa?id=459645432012

Mills, M., Culbertson, S. y Fullagar, C. (2012). Conceptualizing and Measuring Engagement: An Analysis of the Utrech Work Engagement Scale. Journal of Happiness Studies, 13(3), 519545. doi:10.1007/s10902-011-9277-3

Moreno-Jiménez, B., Rodríguez-Carvajal, R. y Escobar, E. (2001). La evaluación del burnout profesional. Factorización del MBI-GS: Un análisis preliminar. Ansiedad y Estrés, 7(1), 69-78. Recuperado de http://www.integraorg.com/wpcontent/docs/La\%20Evaluacion $\% 20$ del $\% 20$ Burnout\%20Profesional.pdf

Moura, D., Orgambídez, A. y Gonçalves, G. (2014). Role Stress and Work Engagement as Antecedents of Job Satisfaction: Results From Portugal. Europe's Journal of Psychology, 10(2), 291-300. doi: 10.5964/ejop.v10i2.714
Oramas, A, González, A. y Vergara, A. (2007). El desgaste profesional. Evaluación y factorización del MBI-GS. Revista Cubana de Salud y Trabajo, 8(1), 37-45. Recuperado de http://www.sld.cu/ galerias/pdf/sitios/insat/rst06107.pdf

Ouweneel, E., Le Blanc, P., \& Schaufeli, W. (2012). Don't Leave your Heart at Home: Gain Cycles of Positive Emotions, Resources, and Engagement at Work. Career Development International, 17(6), 537-556. doi 10.1108/ 13620431211280123

Ouweneel, E., Le Blanc, P., \& Schaufeli, W. (2013). Believe and You Will Achieve: Changes Over Time in Self-Efficacy, Engagement and Performance. Applied Psychology: Helth and Wellbeing, 5(2), 225-247. doi:10.1111/ aphw.12008

Palenzuela, D. L. (1983). Construcción y validación de una escala de Autoeficacia percibida, específica de situaciones académicas. Análisis y Modificación de Conducta, 9(21), 185-219. Recuperado de https://www.researchgate.net/ publication/232599327_Construccion_y_validacion_de_una_escala_de_autoeficacia_percibida_especifica_de_situaciones_academicas_ Construction_and_validation_of_a_scale_of_ perceived_self-sufficiency_for_academic_situations

Paradnikè, K., \& Bandzevičienè, R. (2016). Career Construction in Academic Setting: Links Between Career Adaptability and Study Engagement. International Journal of Psychology: Biopsychosocial Approach, 18, 71-88. doi: 10.7220/2345-024X.18.4

Pujol, L. y Arraigada, M. (2018). Propiedades psicométricas de la versión corta de la Utrecht Work Engagement Scale en trabajadores argentinos. Pensamiento Psicológico, 16(2), 31-45. doi: 1011144/Javerianacali.PPSI16-2.ppvc

Rodríguez, R., Martínez, M. y Sánchez, I. (2014). Análisis de las propiedades psicométricas de la Utrecht Work Engagement Scale en una muestra de trabajadores en Puerto Rico. Universitas Psychologica, 13(4), 1255-1266. doi:10.11144/ Javeriana.UPSY13-4.appu

Sánchez-Cardona, I., Rodríguez-Montalbán, R., Toro-Alfonso, J. y Moreno-Velázquez, I. (2016). Propiedades psicométricas de la 
Utrecht Work Engagement Scale-Student (UWES-S) en universitarios de Puerto Rico. Revista Mexicana de Psicología, 33(2), 121134. Recuperado de http://www.redalyc.org/ articulo.oa?id=243056044004

Schaufeli, W., \& Bakker, A. (2003). Utrecht Work Engagement Scale. Preliminary Manual. Utrecht: Ocupational Health Psychology Unit, Utrecht University.

Schaufeli, W., Martínez, I., Marques-Pinto A., Salanova, M., \& Bakker, A. (2002). Burnout and Engagement in University Students. A Cross-National Study. Journal of CrossCultural Psychology, 33(5), 464-481. doi: 10.1177/0022022102033005003

Seppälä, P., Mauno, S., Feldt, T., Hakanen, J., Kinnunen, U., Tolvanen, A., \& Schaufeli, W. (2009). The Construct Validity of the Utrecht Work Engagement Scale: Multisample and Longitudinal Evidence. Journal of Happiness Studies, 10(4), 459-481. doi: 10.1007/s10902008-9100-y

Simbula, S., Guguelmi. D., Schaufeli, W., \& Depolo, M. (2013). An Italian Validation of the Utrecht Work Engagement Scale: Characterization of Engaged Groups in a Sample of Schoolteachers. Applied Psychology Bulletin, 61(268), 4354. doi: 10.1080/13594320903513916

Uludag, O. (2016). The Mediating Role of Positive Affectivity on Testing the Relationship of Engagement to Academic Achievement: An
Empirical Investigation of Tourism Students. Journal of Teaching in Travel \& Tourism, 16(3), 163-177. doi:10.1080/15313220.2015.11231 30

Vilagut, G., Ferrer, M., Rajmil, L., Rebollo, P., Permanyer-Miralda, G., Quintana, J.,... Alonso, J. (2005). El Cuestionario de Salud SF-36 español: Una década de experiencia y nuevos desarrollos. Gaceta Sanitaria, 19(2), 135-150. Recuperado de http://scielo.isciii. es/scielo.php?script=sci_arttext\&pid=S0213$91112005000200007 \& \operatorname{lng}=\mathrm{es} \& \ln =\mathrm{es}$

Vizoso, C. y Arias, O. (2016). Engagement, burnout y rendimiento académico en estudiantes universitarios y su relación con la prioridad en la elección de la carrera. Revista de Psicología y Educación, 11(1), 45-60. Recuperado de http://www.revistadepsicologiayeducacion.es/ pdf/135.pdf

Ware, J., \& Gandek, B. (1998). Overview of the SF36 Health Survey and the International Quality of Life Assessment (IQOLA) Project. Journal of Clinical Epidemiology, 51(11), 903-912. doi: 10.1016/s0895-4356(98)00081-x

Zecca, G., Györkös, C., Becker, J., Massoudi, K., De Bruin, G., \& Rossier, J. (2015). Validation of the French Utrecht Work Engagement Scale and Its Relationship with Personality Traits and Impulsivity. Revue Européenne de Psychologie Appliquée, 65(1), 19-28. doi:10.1016/j. erap.2014.10.003

Para citar este artículo / To cite this article / Para citar este artigo: Martínez-Uribe, P., Cassaretto-Bardales, M. y Tavera-Palomino, M. (2020). Variables predictoras del compromiso laboral y académico en trabajadores y estudiantes de una universidad peruana. Pensamiento Psicológico, 18(1), 7-19. doi:10.11144/Javerianacali.PPSI18-1.vpcl 